\title{
X-ray Studies on Effect of Annealing on Crystal Structure of Polyethylene Single Crystals
}

\author{
Koh-ichi Iohara, Kiyohisa Imada, and Motowo Takayanagr \\ Department of Applied Chemistry, Faculty of Engineering, Kyushu University, \\ Fukuoka, Japan
}

(Received March 10, 1972)

\begin{abstract}
Effect of annealing on the crystal structure of polyethylene single crystals was studied by using X-ray diffraction method. The dimension of $a$ axis, the unit-cell volume, and the disorder factor which represents the mean square displacements of carbon atoms from their average locations were found to increase with increasing annealing temperature up to $105^{\circ} \mathrm{C}$, below which no lamellar thickening was observed. On the other hand, their values turned to show a decreasing tendency with increasing annealing temperatures above $105^{\circ} \mathrm{C}$, where lamellar thickening by annealing was observed. Increase of the unit-cell volume and the disorder factor can be explained by an increase of population of crystal defects introduced into the crystal by thermal motions of molecular chains largely amplified at sufficiently high temperatures. The abnormal structure generated by the rotation of molecular chains around their axes by about $90^{\circ}$ was assumed as one of the crystal defects. A fraction of the abnormal structure was estimated by the method previously developed by us on the X-ray diffraction intensities. In the case of the single crystals annealed at $120^{\circ} \mathrm{C}$, this amounted to $2 \%$ of the whole crystal.
\end{abstract}

KEY WORDS X-ray / Cell Dimension / Disorder Factor / Crystal Defect / Rotational Transition / Annealing / Single Crystal / Polyethylene /

There have been many measurements made on the effects of annealing on the structure and properties of polyethylene single crystals. It is well known that annealing increases the lamellar thickness of the crystal. At the same time, some authors have pointed out that the heat treatment of single crystals introduces defects into the crystal and distorts the lattice regularity from results based on measurements of mechanical properties ${ }^{1}$, nuclear magnetic resonance $^{2-4}$ and electron spin resonance ${ }^{4}$, and the observation of moiré pattern of bilayered crystals by using an electron microscope $e^{5-7}$. Increase of lamellar thickness and generation of crystal defects are expected to have some influence on the unit cell dimensions and the disorder factor.

Concerning this subject, Eby, et al. ${ }^{8-10}$ reported the annealing effect on the cell dimensions of single crystals and bulk crystallized samples of polyethylene. They found that annealing reduced the unit cell volume of the crystal, and explained their results as due to the lamellar thickening. However, they did not clarify the influence of the crystal defects introduced by annealing on the lattice dimensions. We will take up this subject in this paper.

In a previous paper $^{11}$, we have developed a method for evaluating the fraction of molecular chains rotated by an angle of $90^{\circ}$ from the normal orientation. This method will be used in this paper to discuss the effect of defects associated with the rotational transition of molecular chains on the structure and properties of the crystals.

It should be noted here that the lamellar crystals in the bulk crystallized sample are rather properly considered to correspond with the annealed single crystals in their structure and properties ${ }^{1}$. We always take the as-grown single crystals as the starting material and examine the annealing effect on such materials. 


\section{EXPERIMENTAL}

The sample used in this study was a commercial high density linear polyethylene, Hizex 2200J, the product of Mitsui Petrochemical Industries, Co., Ltd. Its molecular weight $\left(M_{v}\right)$ was 40000 , and the degree of branching was 3$\mathrm{CH}_{3} / 1000 \mathrm{CH}_{2}$.

Single crystals were prepared by gradually dropping a hot concentrated xylene solution of polyethylene to xylene, which was kept at the crystallization temperature $80^{\circ} \mathrm{C}$, to finally form a $0.05-\%$ solution. After this operation, single crystals were isothermally crystallized for $24 \mathrm{hr}$, which are designated here in as-grown single crystals. Some of the single crystals were filtered and formed into oriented mats. Other ones, on the other hand, were filtered to form unoriented aggregates of crystal lamellae. Orientations of crystal lamellae in these samples were confirmed by the X-ray diffraction method. After they were dried under vacuum, heat treatment was affected by heating the as-grown single crystals wrapped in a metal foil in an oil bath for $20 \mathrm{hr}$.

Unit cell dimensions, $a$ and $b$, were determined by the diffraction angles of three Bragg reflections, (110), (200) and (020) by using an $\mathrm{X}$-ray diffractometer with Geiger-Müller counter. Quartz was used to correct the diffraction angle. Measurements of thermal expansion of unit cell dimensions were conducted by using a low temperature device of Rigakudenki Company.

The disorder factors, which represent the mean square displacements of carbon atoms from their average locations, were determined especially for the unoriented samples based on the diffraction intensities of 24 Bragg reflections according to the procedure reported previous$1 y^{12}$.

Long periods of the single crystal mats were determined by the small angle X-ray scattering method.

\section{RESULTS}

\section{Effect of Annealing on Unit-Cell Dimensions}

Figure 1 shows the unit-cell dimensions, $a, b$, and $c$, and the unit-cell volume, $V$, as a func-

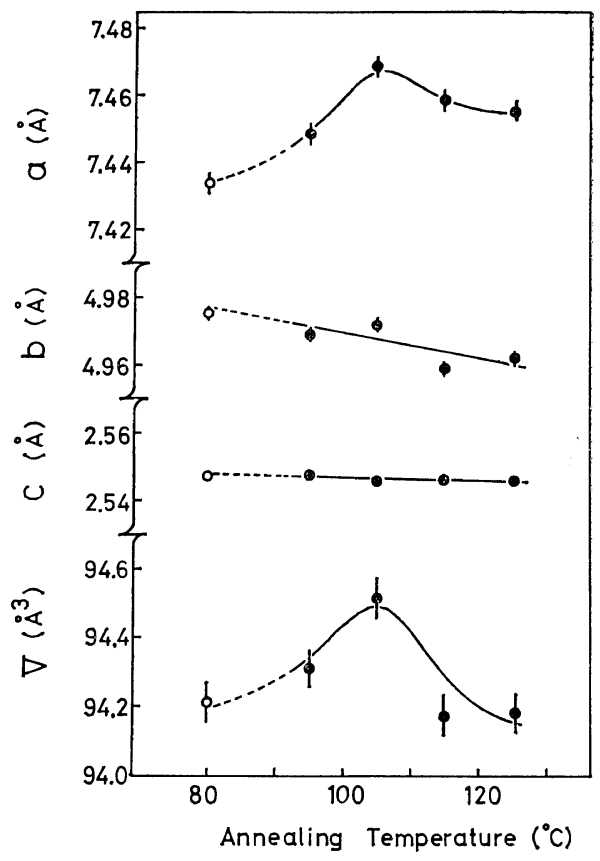

Figure 1. Unit-cell dimensions, $a, b$, and $c$, and unit-cell volume vs. annealing temperature:

$O$, as-grown single crystal prepared at $80^{\circ} \mathrm{C}$;

, annealed single crystal.

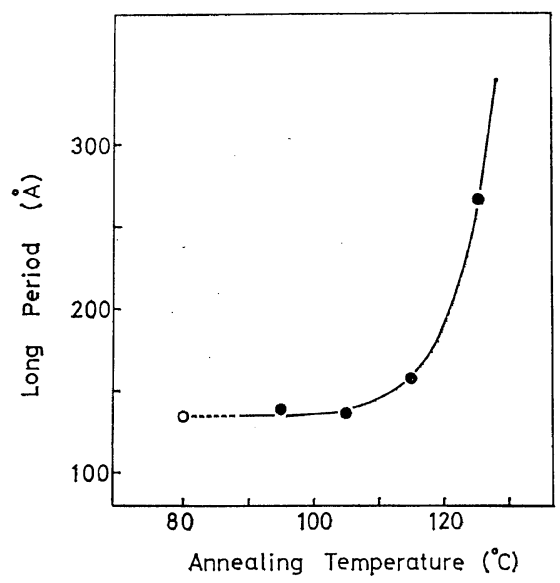

Figure 2. Long period vs. annealing temperature: $O$, as-grown single crystal prepared at $80^{\circ} \mathrm{C}$; - annealed single crystal.

tion of annealing temperature. The measurements were made at $18^{\circ} \mathrm{C}$. The standard deviations of the values of $a, b$, and $c$ were about $0.003,0.002$, and $0.001 \AA$, respectively, and that of $V$ was estimated to be about $0.06 \AA^{3}$. The 
crystals annealed at relatively low temperatures below $105^{\circ} \mathrm{C}$ show increasing tendencies of both the $a$ dimension and the unit-cell volume and a decreasing tendency of the $b$ dimension with increasing annealing temperature. At the temperatures below $105^{\circ} \mathrm{C}$, the increase of lamellar thickness by annealing is negligible as shown in Figure 2. On the other hand, when the samples are annealed at relatively high temperatures above $105^{\circ} \mathrm{C}$, the expansions of $a$ and $V$ show decreasing tendencies from the values at $105^{\circ} \mathrm{C}$ with increasing annealing temperature. At the same time, the lamellar thickness shows a rapid increase by annealing at these higher temperatures as seen in Figure 2. Thus, the curves of $a$ and $V$ against annealing temperature show the maxima at about $105^{\circ} \mathrm{C}$, at which the lamellar thickening is initiated.

\section{Effect of Annealing on Disorder Factor}

Figure 3 shows the disorder factors of the asgrown and annealed single crystals. The disorder factors which are caused by the molecular vibrations and the crystal imperfections of the first kind $^{12}$, are represented by two elements,

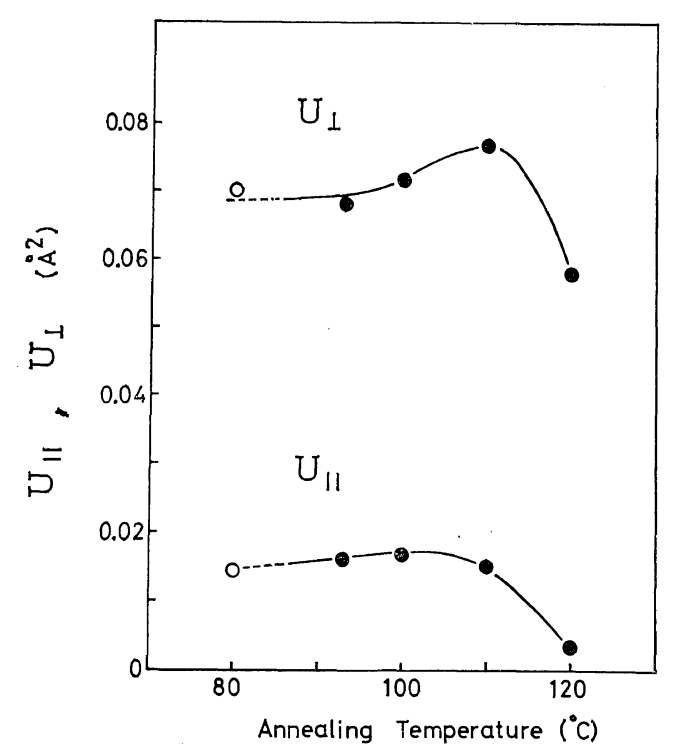

Figure 3. Disorder factors, $U_{\|}$and $U_{\perp}$, vs. annealing temperature. $U_{\|}$and $U_{\perp}$ indicate mean square displacements of carbon atom in directions parallel and perpendicular to molecular axis:

$\bigcirc$, as-grown single crystal prepared at $80^{\circ} \mathrm{C}$;

, annealed single crystal.
$U_{\|}$and $U_{\perp}$, along the directions parallel and perpendicular to the chain axis, respectively. The standard deviations of $U_{\|}$and $U_{\perp}$ due to the fluctuations of the observed X-ray intensity data, are about $0.002 \AA^{2}$ and $0.003 \AA^{2}$, respectively. The disorder factors seem to slightly increase with increasing annealing temperatures except those of the sample annealed at $120^{\circ} \mathrm{C}$, in which both values are definitely decreased.

\section{DISCUSSION}

\section{Factors of Annealing Regulating Cell Dimensions and Disorder Factors}

The results shown in Figures $1-3$ suggest that annealing has two opposite influences on the crystal. One effect is to expand the $a$ dimension and the unit-cell volume, and increase the disorder factors. This effect is remarkable in annealing even at the low temperatures, where lamallar thickening is negligible. The other effect is to decrease the unit-cell volume and the disorder factors. The latter effect is observable in annealing at the relatively high temperatures, where lamellar thickening is accompanied by annealing. The latter effect of annealing is closely related to the thickness of the lamella, which has already been discussed by Eby, et $a .^{8-10}$. In this study, therefore, the former effect will be discussed. Hereafter, annealing effect is used as the meaning of the former annealing effect.

Eby, et al.,$^{8}$ have found the former annealing effect on the cell dimensions, but they attributed it to the mechanical deformation of the asgrown single crystals during their packing process into capillaries by using a hypodermic syringe. In the sample preparation method employed in this study, there were no circumstances to effect any mechanical distortion on the crystals except during the process of filtration of the single crystal suspensions. In order to check this possibility, the unit-cell dimensions were reexamined for the samples prepared by the following methods. In one method, xylene was evaporated in vacuum without separating the crystals by filtrations, and the single crystal cakes were gathered from the bottom of the flask with a spatula. In the other method, xylene was replaced with meth- 
anol and the latter was evaporated at the normal pressure and temperature. Such procedures clarified that all of these samples prepared without being filtrated showed the same behavior as those prepared by the filtration process as far as the annealing effect was concerned. From these experiments, the variation of the cell dimensions by annealing in this study can not be ascribed, at least, to any accidental deformation of the crystals during the handling of the samples. Thus, we consider the phenomena as the ones characteristic of the as-grown single crystals which have never been heat-treated at the temperatures higher than those of crystallization.

It should be noted here that the unit-cell volume and the disorder factors increase upon annealing at low temperatures. These results indicate that the crystals are disordered upon annealing, and are consistent with the results obtained from other kinds of measurements. According to the measurements of viscoelastic properties on single crystal mats ${ }^{1}$, intensity of crystalline absorption was decreased upon annealing. The observation by electron microscope $^{5-7}$ have reported that moiré pattern of bilayered single crystals was distorted by annealing at the temperatures a few degree higher than that of crystallization although lamellar thickness of the single crystals was not increased.

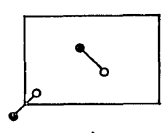

A

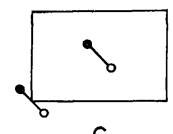

C

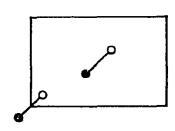

B

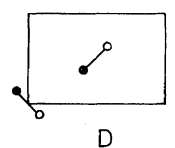

D
Figure 4. Unit-cell structures of polyethylene crystal by taking account of the rotational transition of a molecular chain:

A, normal structure;

$\mathrm{B}, \mathrm{C}$, and $\mathrm{D}$, abnormal structure.

One chain rotates around its chain axis $c a .90^{\circ}$ and transforms to the abnormal orientation in $B$ and C. Both chains are in the abnormal state in D.
All these results were interpreted to indicate that defects were introduced into crystals and lattice regularity was distorted by annealing. The defects have been discussed on the basis of their experiments. In the following section, we will treat the defects based on X-ray diffraction method.

Interpretation of Annealing Effect based on Rotational Transition of Molecules

As one of the disorders in the crystal, we considered in a previous paper ${ }^{11}$ the abnormal structures B, C and D as shown in Figure 4, which were generated by the rotational transition of one or two of the two chains in the normal structure A from the normal orientation to the abnormal orientation by the rotation around their chain axes by about $90^{\circ}$. According to the calculation of the average structure factor of X-ray diffraction for the crystal including the normal and abnormal structures, a fraction of the abnormal structures in the crystal can be evaluated by examining the relative value of the integrated intensities of $h k l$ reflections with $h+k=$ odd to those of reflections with $h+$ $k=$ even, that is, $\sum w_{\mathrm{j}} I_{\mathrm{j}}(h+k=\mathrm{odd}) / \sum w_{\mathrm{j}} I_{\mathrm{j}}(h+$ $k=$ even), where $I_{\mathrm{j}}$ and $w_{\mathrm{j}}$ are integrated intensity and weighting factor of each reflection. This relative value should decrease with increasing fraction of the abnormal structures.

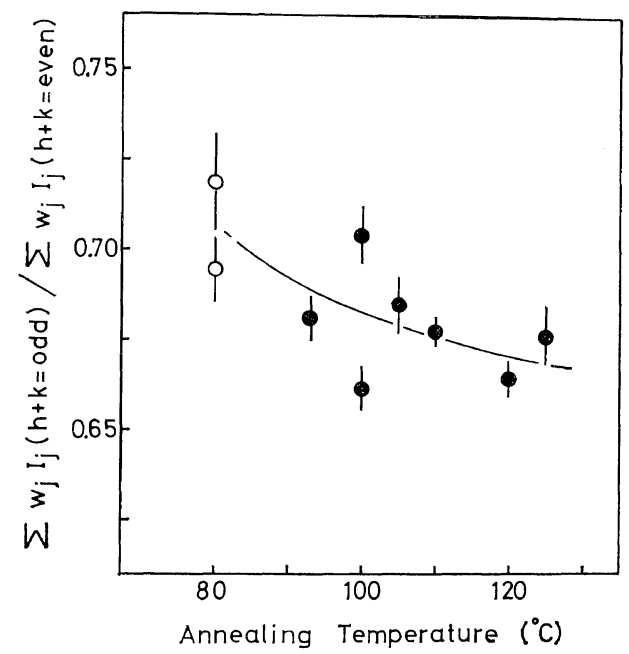

Figure 5. Relative value vs. annealing temperature: $\bigcirc$, as-grown single crystal prepared at $80^{\circ} \mathrm{C}$;, annealed single crystal. 
The relative values were estimated for the asgrown and annealed single crystals, using the diffraction data at $12^{\circ} \mathrm{C}$. The intensities of 210 , $120,011,211,121,410$, and 320 reflections and those of $020,310,111,201,220,400$, and 311 reflections were used in the estimation, and the reciprocal of the standard deviation of each observed intensity was adopted as the weight of each reflection, $w_{j}{ }^{14}$. Figure 5 shows the relative values at $12^{\circ} \mathrm{C}$ as a function of annealing temperature. The fluctuation of the data is somewhat large, but a decreasing tendency with increasing annealing temperature is observable.

If the as-grown single crystal is assumed to be in the completely ordered state consisting of the normal structure, a fraction of polyethylene chains in the abnormal orientation is estimated to be about $1 \%$ of the whole molecules in the single crystal annealed at $120^{\circ} \mathrm{C}$. Then, the total fraction of the abnormal structures $\mathrm{B}, \mathrm{C}$ and $\mathrm{D}$ retained in this annealed sample is estimated to be about $2 \%$ of the whole crystal.

In a previous paper ${ }^{11}$, it was reported that the polyethylene chains in the crystal showed an increasing tendency of the rotational transition with increasing ambient temperature above $0^{\circ} \mathrm{C}$. A fraction of chains in the abnormal orientation at $120^{\circ} \mathrm{C}$ was estimated to be about $5 \%$ of the whole molecules.

By considering the above results, increases of the abnormal structures in the annealing single crystals may be explained as follows. Molecules in the crystal are disordered according to the thermodynamic requirement at the high annealing temperatures, and some chains are transformed to the abnormal state due to largely amplified rotational motions around the molecular axes. When they are cooled down to room temperature, a portion of the rotated chains are frozen in as one kind of crystal defects, although most of them might return to the original ordered state.

These retained abnormal structures may have a tendency to deform themselves toward the energetically more stable structures, whereas such a deformation is forbidden by the surrounding crystal field consisting of the normal structure. However, this tendency of the retained abnormal structures may more or less distort the crystal lattice, and give rise to the increase of the unit-cell volume and the disorder factor. Thus, the former annealing effect is explained qualitatively. The results shown in Figures 1 and 3 that $a$ dimension, unit-cell volume and $U_{\perp}$ show the maxima are explained by increasing fraction of the retained abnormal structures after cooling from the annealing temperatures above $80^{\circ} \mathrm{C}$ and the increasing lamellar thickness of the crystals annealed above $105^{\circ} \mathrm{C}$. The last factor of increased lamellar thickness plays a role of stabilizing the crystal structure, as Eby, et al., ${ }^{8-10}$ have already mentioned.

Acknowledgement. The authors express their sincere gratitude to Mr. Suehiro of this laboratory for his useful discussion and helpful technical advice.

\section{REFERENCES}

1. M. Takayanagi and T. Matsuo, J. Macromol. Sci., B1, 407 (1967).

2. W.P. Slichter, J. Appl. Phys., 31, 1865 (1961).

3. W. R. Kedzie, cited by P. H. Geil, "Polymer Single Crystals," Wiley Interscience, New York, N.Y. 1963.

4. N. Kusumoto, T. Yamamoto, and M. Takayanagi, J. Polym. Sci., Part A-2, 9, 1173 (1971).

5. V.F. Holland, J. Appl. Phys., 35, 3235 (1964).

6. D.C. Bassett, Phil. Mag., 17, 37 (1968).

7. K. Abe, M. Niinomi, and M. Takayanagi, $J$. Macromol. Sci. B4, 87 (1970).

8. G. T. Davis, R.K. Eby, and G. M. Martin, J. Appl. Phys., 39, 4973 (1968).

9. G.T. Davis, R.K. Eby, and J.P. Colson, ibid., 41, 4316 (1970).

10. R. K. Eby and J.P. Colson, ibid., 41, 4313 (1970).

11. K. Iohara, K. Imada, and M. Takayanagi, Polymer $J$., in press.

12. K. Iohara, K. Imada, and M. Takayanagi, Polymer J., 3, 357 (1972).

13. M. Takayanagi, T. Aramaki, M. Yoshino, and K. Hoashi, J. Polym. Sci., 46, 531 (1960).

14. "X-sen Kesshōgaku (X-ray Crystallography)," Vol. 2, Y. Nitta, Ed., Maruzen Co., Ltd., Tokyo, 1961, Chapter 3. 